\title{
Síndrome de takotsubo secundario a traumatismo raquimedular
}

\section{María Ortiz-Lasa*, Alejandro González-Castro, Yhivian Peñasco-Martín, Marta López-Sánchez y Olazt Azcune-Echeverría}

\section{Hospital Universitario Marqués de Valdecilla, Unidad de Cuidados Intensivos, Santander, Cantabria, España}

Recibido el 15 de febrero de 2017; aceptado el 13 de julio de 2017

Disponible en Internet el 23 de febrero de 2018

\section{PALABRAS CLAVE}

Takotsubo;

Miocardiopatía por

estrés;

Traumatismo

raquimedular

\section{KEYWORDS}

Takotsubo;

Stress

cardiomyopathy;

Spinal cord injury

\begin{abstract}
Resumen
Antecedentes: El síndrome de takotsubo secundario a traumatismo raquimedular cervical es infrecuente y no se describen series de casos en la literatura.

Pacientes y método: Se describe el caso clínico de una mujer de 82 años que ingresó en la Unidad de Cuidados Intensivos tras traumatismo raquimedular cervical y como consecuencia desarrolló miocardiopatía de takotsubo.

Resultado: Desarrollo de una miocardiopatía de takotsubo tras un traumatismo raquimedular cervical.

Conclusiones: En la actualidad el diagnóstico de miocardiopatía de takotsubo en Cuidados Intensivos está en aumento, en parte por el uso de la ecocardiografía trastorácica por parte de los Intensivistas; con ello se descartan otras causas posibles de la misma y no sólo la cardiológica o la descarga catecolaminérgica secundaria a una situación de estrés.

(c) 2017 Sociedad Colombiana de Cardiología y Cirugía Cardiovascular. Publicado por Elsevier España, S.L.U. Este es un artículo Open Access bajo la licencia CC BY-NC-ND (http:// creativecommons.org/licenses/by-nc-nd/4.0/).
\end{abstract}

\section{Takotsubo syndrome secondary to spinal cord injury}

\section{Abstract}

Background: Takotsubo syndrome secondary to spinal cord injury is rare, and there are no case series described in the literature.

Patients and method: A clinical case is presented of an 82 year-old woman admitted to the Intensive Care Unit after a spinal cord injury, and as a results developed Takotsubo cardiomyopathy.

\footnotetext{
* Autor para correspondencia.

Correo electrónico: maralasa111@gmail.com (M. Ortiz-Lasa).
} 
Results: A Takotsubo cardiomyopathy developed after a spinal cord injury.

Conclusions: The diagnosis of Takotsubo cardiomyopathy is currently increasing in Intensive Care Units. This is partly due to use of transthoracic echocardiography by intensive care specialists. Using this technique they can rule out other possible causes of this condition, and not just the cardiological ones, or the catecholamine release following a stressful event.

(c) 2017 Sociedad Colombiana de Cardiología y Cirugía Cardiovascular. Published by Elsevier España, S.L.U. This is an open access article under the CC BY-NC-ND license (http:// creativecommons.org/licenses/by-nc-nd/4.0/).

\section{Introducción}

Han transcurrido 26 años desde que Sato et al. ${ }^{1}$ describieron un síndrome caracterizado por dolor torácico con cambios electrocardiográficos y bioquímicos similares a un infarto agudo de miocardio, con alteraciones de la contractilidad apical del ventrículo izquierdo, pero con arterias coronarias angiográficamente normales, conocido como síndrome de takotsubo o miocardiopatía de estrés. Las enfermedades del sistema nervioso central (hemorragia subaracnoidea, epilepsia, ictus isquémico) se han reconocido como desencadenantes de dicho síndrome ${ }^{2}$, sin embargo es excepcional su presencia luego de un traumatismo raquimedular, y por tanto un pequeño número de casos descritos en la literatura $^{3-6}$. Se ilustra el caso de una mujer de 82 años que tras traumatismo raquimedular cervical desarrolló miocardiopatía de takotsubo.

\section{Caso}

Se trata de una mujer de 82 años, sin antecedentes personales de interés, que ingresó en la Unidad de Cuidados Intensivos de un hospital de tercer nivel de atención, tras ser atendida en el Servicio de Urgencias, por traumatismo múltiple debido a una caída fortuita. Como consecuencia, sufrió traumatismo craneoencefálico leve, traumatismo facial complejo con fractura de huesos propios de la nariz, hueso maxilar y pared lateral de la órbita izquierda, además de fractura de C1-C2 inestable con invasión de canal medular. Al ingreso en la Unidad de Cuidados Intensivos, se le asignó un valor en la escala de Glasgow de 14, con tendencia al sueño y sin focalidad neurológica. En un período de 30 minutos desarrolló insuficiencia respiratoria secundaria a broncoaspiración por epistaxis importante y claudicación respiratoria, por lo que se decidió intubación orotraqueal. De forma concominante, en el monitor se observó elevación progresiva del ST, por lo cual se hizo de inmediato un electrocardiograma que reflejó elevación del segmento ST en V4-V6 sin otras alteraciones visibles (fig. 1) y se determinaron las enzimas cardíacas: troponina inicial de $0 \mathrm{ng} / \mathrm{ml}$. Así mismo, se practicó ecocardiografía transtorácica, en la que observó "ballooning" apical del ventrículo izquierdo con discinesia apical e hipercontractilidad de los segmentos basales (fig. 2). Fue valorada por el servicio de Cardiología Críticos del hospital y dada su situación general, se desestimó la realización de coronariografía como prueba confirmatoria. Una vez valorado el riesgo/beneficio, con los datos electrocardiográficos y ecocardiográficos se asumió que se trataba de un síndrome de takotsubo. En el transcurso de las

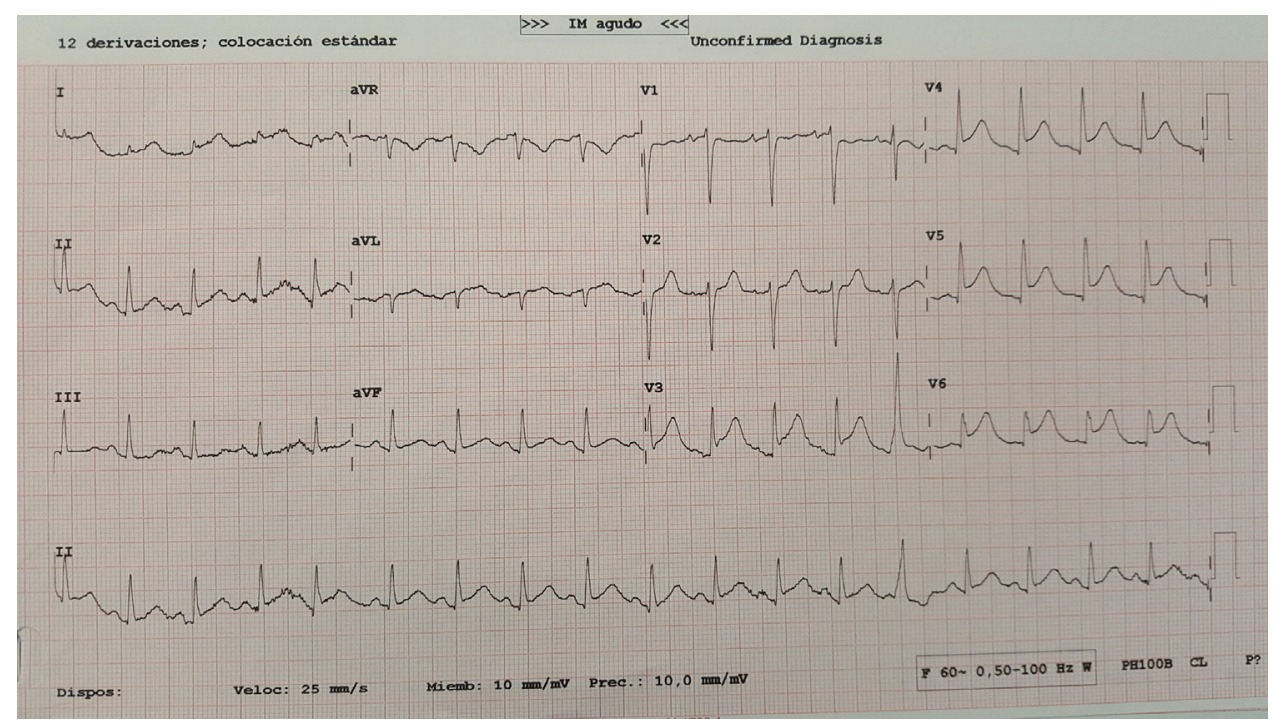

Figura 1 Electrocardiograma inicial. 


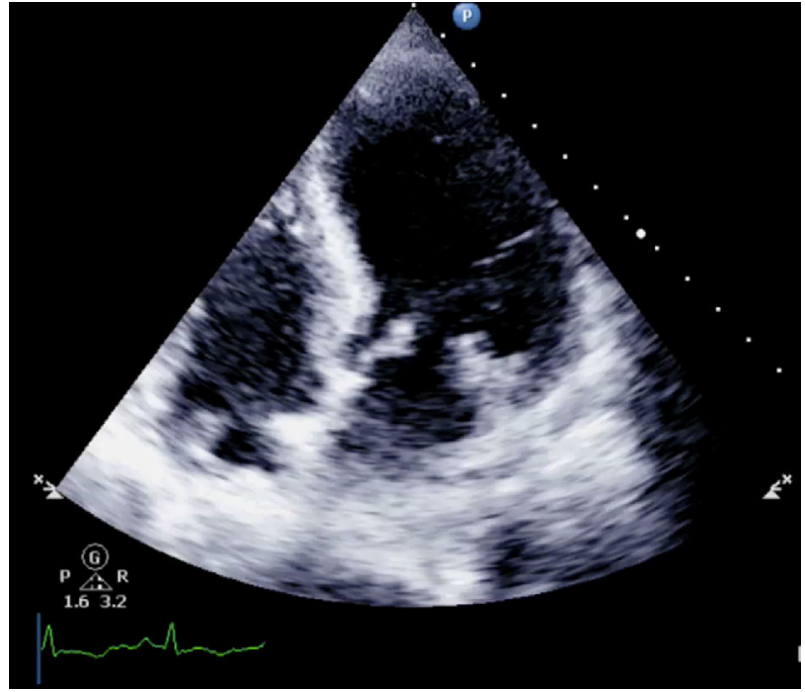

Figura 2 Ecocardiograma inicial.

24 horas posteriores el electrocardiograma demostró un descenso de las onda T en V4-V6 (fig. 3) y elevación de la troponina hasta alcanzar un pico máximo de $0,86 \mathrm{ng} / \mathrm{ml}$. A las 48 horas se repitió el ecocardiograma y se evidenció FEVI $30 \%$ con acinesia apical e hiperkinesia de segmentos basales. Fue trasladada a planta tras cinco días de ingreso en nuestra Unidad. Al décimo día de ingreso se ordenó un nuevo ecocardiograma en el que se confirmó la reversibilidad de las alteraciones de la contractilidad observadas inicialmente, además de mejoría de la acinesia apical e hiperkinesia de segmentos basales y FEVI $50 \%$ sin complicaciones.

\section{Discusión}

El síndrome takotsubo, conocido también como miocardiopatía inducida por estrés, síndrome de discinesia apical transitoria, aturdimiento miocárdico o balonamiento apical del ventrículo izquierdo, fue visto por primera vez en Japón, por Sato et al. ${ }^{1}$, en 1990, quienes lo describieron como un cuadro clínico conformado por dolor torácico con cambios electrocardiográficos y bioquímicos que imitaban a un infarto agudo de miocardio, con alteraciones de la contractilidad apical del ventrículo izquierdo, pero con arterias coronarias angiográficamente normales, donde radica la principal diferencia con el infarto agudo de miocardio. Posteriormente, en 2001, Tsuchihashi et al. ${ }^{7}$ analizaron una serie de 88 pacientes que cumplían criterios de discinesia transitoria del ventrículo izquierdo con arterias coronarias angiográficamente normales y sin miocardiopatías conocidas. En su serie, el $86 \%$ fueron mujeres y en el $43 \%$ se objetivó agravamiento de una enfermedad subyacente ( 3 casos de ataque cerebrovascular, 3 de epilepsia, 3 de exacerbación asmática, 7 de abdomen agudo y 11 de cirugía) y el $27 \%$ sufrió problemas psíquicos y emocionales. En 2014 se publicaron los datos del estudio $\mathrm{RETAKO}^{8}$, el registro nacional en España del síndrome de takotsubo, en el que durante un período de 2 años se incluyeron 202 pacientes, $90 \%$ de los cuales eran mujeres. Como en la serie de Tsuchihashi, existe un número de casos acompañados de agravamiento de enfermedades subyacentes $(20 \%)$, en tanto que el estrés psicológico aislado se observó hasta en un 50\%. En ninguna de las dos series se detecta concretamente el traumatismo raquimedular como desencadenante del síndrome. En la literatura hay pocos casos descritos de traumatismo como desencadenante del síndrome en mención, entre los que se hallan los descritos por Riera, Vergez, Morita y Krpata ${ }^{3-6}$ (tabla 1).

Su incidencia es baja, en torno al 1-2\% de todos los casos que ingresan por sospecha de infarto agudo de miocardio. En los últimos datos publicados por el registro internacional de takotsubo se determinó que la edad media de presentación oscila entre los 53 y 79 años 9 . Si bien la causa del síndrome está lejos de ser esclarecida, sí se han

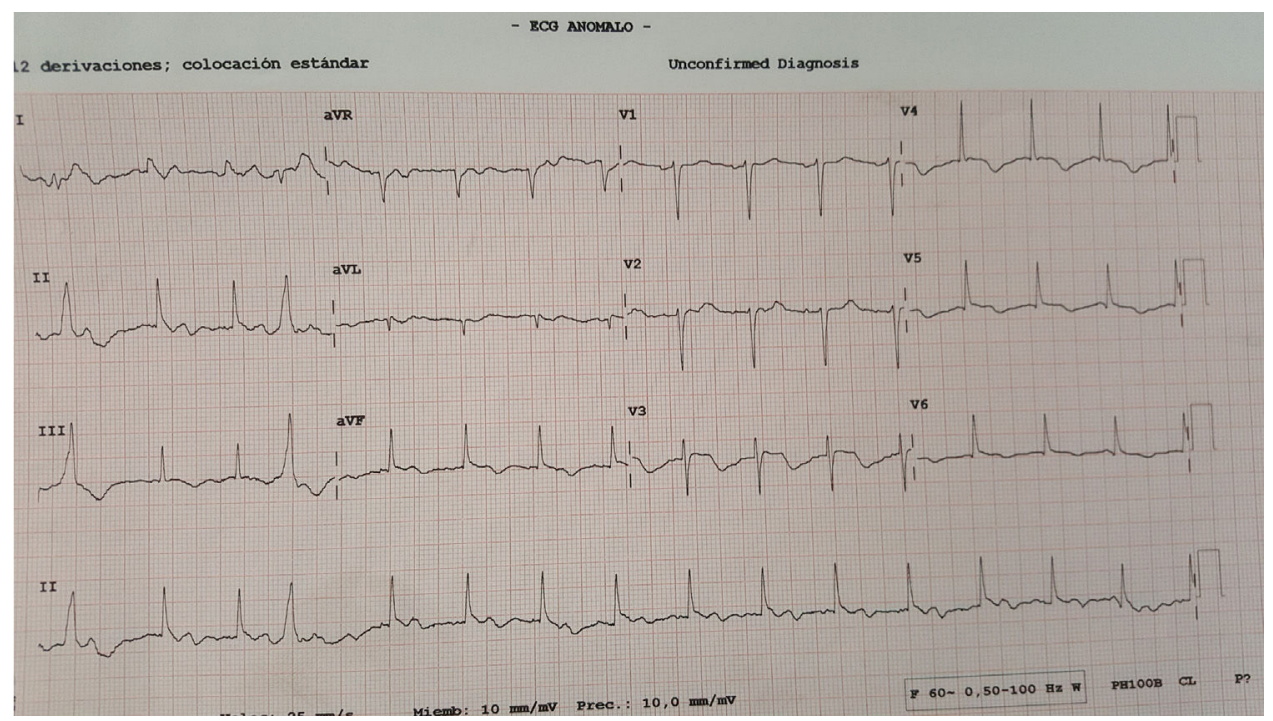

Figura 3 Electrocardiograma evolutivo. 
Tabla 1 Descripción de la cohorte de enfermos diagnósticados de síndrome de takotsubo secundario a traumatismo

\begin{tabular}{|c|c|c|c|c|c|c|c|c|c|}
\hline Casos & Sexo & $\begin{array}{l}\text { Edad } \\
\text { (años) }\end{array}$ & Antecedentes & $\begin{array}{l}\text { Factor } \\
\text { desencade- } \\
\text { nante }\end{array}$ & $\begin{array}{c}\text { Tiempo de } \\
\text { aparición del } \\
\text { síndrome desde el } \\
\text { ingreso (horas) }\end{array}$ & $\begin{array}{c}\text { Características } \\
\text { ECG }\end{array}$ & $\begin{array}{c}\text { s Características } \\
\text { ETE }\end{array}$ & $\begin{array}{l}\text { Necesidad de } \\
\text { fármacos } \\
\text { inotrópicos/ } \\
\text { vasopresores }\end{array}$ & $\begin{array}{l}\text { Tiempo de mejoría } \\
\text { de la recuperación } \\
\text { de la disfunción } \\
\text { (días) }\end{array}$ \\
\hline Caso 3 (5) & Hombre & 69 & $\begin{array}{l}\text { NO } \\
\text { disponible }\end{array}$ & $\begin{array}{l}\text { Traumatismo } \\
\text { por caída }\end{array}$ & 12 & $\begin{array}{ll}\text { Elevación } & \\
\text { del ST V2-V5 } & \text { S }\end{array}$ & $\begin{array}{l}\text { Disfunción } \\
\text { sistólica moderada } \\
\text { y aquinesia apical }\end{array}$ & NO & 4 \\
\hline
\end{tabular}


postulado diferentes hipótesis. Inicialmente se consideró que el espasmo multivaso era el causante de la discinesia transitoria del ventrículo izquierdo, pero sólo ha llegado a demostrarse mediante pruebas de provocación en menos del $30 \%$ de los casos. Posteriormente se describieron mecanismos numerosos, entre los que se destacan miocarditis, rotura de placas no obstructivas con trombólisis espontánea, alteraciones microvasculares, alteraciones anatómicas como arteria descendente anterior muy larga y sepsis. En la actualidad, el de mayor aceptación es la toxicidad por niveles elevados de catecolaminas y neuropéptidos de estrés. Los criterios diagnósticos en los que se basan la mayoría de los autores para el diagnóstico son los establecidos por la clínica Mayo, modificados en 2008, entre los que se encuentran:

- Alteraciones transitorias en la contractilidad ventricular izquierda (discinesia, acinesia o hipocinesia), con afectación apical o sin ella, extendiéndose más allá del territorio de una arteria coronaria determinada; ocasionalmente hay una situación estresante desencadenante, aunque puede no estar presente.

- Ausencia de enfermedad coronaria obstructiva o evidencia angiográfica de rotura de placa aguda.

- Nuevas alteraciones electrocardiográficas (elevación del ST y/o inversión de la onda T) o elevación modesta de troponina.

- Ausencia de feocromocitoma o miocarditis ${ }^{10}$.

Aunque en general el pronóstico es bueno, las formas secundarias de takotsubo podrían tener peor pronóstico en términos de mortalidad, recurrencias y readmisiones.

No hay recomendaciones claras respecto al tratamiento. Éstas se centran en aplicar el manejo habitual de los síndromes coronarios agudos.

\section{Conclusiones}

En la actualidad el diagnóstico de miocardiopatía de tako-tsubo en Cuidados Intensivos está en aumento, en parte tal vez por el uso creciente de la ecocardiografía trastorácica por los Intensivistas. Para finalizar, se destaca que el síndrome de takotsubo desencadenado por un traumatismo en general y más concretamente por un traumatismo facial y raquimedular es poco frecuente y puede llegar a desempeñar un papel más relevante en un futuro.

\section{Conflictos de interés}

Ninguno.

\section{Bibliografía}

1. Sato H, Tateishi H, Uchida T. Takotsubo type cardiomyopathy due to multivessel spasm. En: Kodama K, Haze K, Hon M, editores. Clinical aspect of myocardial injury: from ischemia to heart failure. Tokyo: Kagaku Hyoronsha; 1990. p. 56-64.

2. Finsterer J, Stöllberger C. Central nervous system disease triggering takotsubo syndrome. Int Cardiovasc Forum J. 2016;5:36-9.

3. Riera M, Llompart-Pou JA, Carrillo A, Blanco C. Head injury and inverted Takotsubo cardiomyopathy. J Trauma. 2010;68:13-5.

4. Vergez M, Pirrachio R, Mateo J, Payen D, Cholley B. Tako Tsubo cardiomyopathy in a patient with multiple trauma. Resuscitation. 2009;80:1074-7.

5. Morita S, Inokuchi S, Yamagiwa T, Aoki H, Nakagawa Y, Yamamoto I. Tako-tsubo-like left ventricular dysfunction with ST-segment elevation after central spinal cord injury: a case report. J Emerg Med. 2008;39:301-4.

6. Krpata D, Edward M, Barksdale Jr. Trauma induced left ventricular apical ballooning syndrome in a 15 year old: a rare case of Tako-tsubo cardiomyopathy. J Pediatric Surg. 2013;48:876-9.

7. Tsuchihashi K, Ueshima K, Uchida T, Ohmura N, Kimura K, Owa $\mathrm{M}$, et al. Transient left ventricular apical ballooning without coronary artery stenosis: a novel heart syndrome mimicking acute myocardial infarction. J Am Coll Cardiol. 2001;38:11-8.

8. Núñez I, Andrés $M$, Almendro $M$, Sionis $A$, Martín A, Bastante $\mathrm{T}$, et al. Caracterización del síndrome de tako-tsubo en España: resultados del registro nacional RETAKO. Rev Esp Cardiol. 2015;68:505-12.

9. Templin C, Ghadri J, Diekmann J, Napp L, Bataiosu DR, Jaguszewshi M. Clinical features and outcomes of takotsubo (stress) cardiomyopathy. N Engl J Med. 2015;373:929-38.

10. Nuñez I, Luaces M, Garcia JC. Cardiopatía de estrés o síndrome de Tako-Tsubo: conceptos actuales. Rev Argent Cardiol. 2009;77:218-23. 